Please do not remove this page

RMIT

UNIVERSITY

\title{
Elastomer-based pneumatic switch for radio frequency microdevices
}

Shah, Charan Manish; Sriram, Sharath; Bhaskaran, Madhu; Nasabi, Mahyar; Nguyen, Giang Thach; Rowe, Wayne; Mitchell, Arnan

https://researchrepository.rmit.edu.au/esploro/outputs/9921858402601341/filesAndLinks?institution=61RMIT_INST\&index=null

Shah, C. M., Sriram, S., Bhaskaran, M., Nasabi, M., Nguyen, G. T., Rowe, W., \& Mitchell, A. (2012).

Elastomer-based pneumatic switch for radio frequency microdevices. IEEE Journal of

Microelectromechanical Systems, 21(6), 1410-1417. https://doi.org/10.1109/JMEMS.2012.2208220

Document Version: Accepted Manuscript

Published Version: https://doi.org/10.1109/JMEMS.2012.2208220

Repository homepage: https://researchrepository.rmit.edu.au

(C) 2012 IEEE

Downloaded On 2023/04/26 22:24:50 +1000

Please do not remove this page 
Thank you for downloading this document from the RMIT Research Repository.

The RMIT Research Repository is an open access database showcasing the research outputs of RMIT University researchers.

RMIT Research Repository: http://researchbank.rmit.edu.au/

\section{Citation:}

Shah, C, Sriram, S, Bhaskaran, M, Nasabi, M, Nguyen, G, Rowe, W and Mitchell, A 2012, 'Elastomer-based pneumatic switch for radio frequency microdevices', IEEE Journal of Microelectromechanical Systems, vol. 21, no. 6, pp. 1410-1417.

See this record in the RMIT Research Repository at:

http://researchbank.rmit.edu.au/view/rmit:17874

Version: Accepted Manuscript

Copyright Statement: (C) 2012 IEEE

Link to Published Version:

http://dx.doi.org/10.1109/JMEMS.2012.2208220 


\title{
Elastomer-based Pneumatic Switch for Radio Frequency Micro-Devices
}

\author{
Charan M. Shah, Sharath Sriram, Member, IEEE, Madhu Bhaskaran, Member, IEEE, Mahyar Nasabi, \\ Thach G. Nguyen, Wayne S. T. Rowe, Member, IEEE, and Arnan Mitchell, Member, IEEE
}

\begin{abstract}
This article reports the realization and characterization of a pneumatic micro-switch integrated with a high frequency RF transmission line on an elastomer substrate. A process for the fabrication of low-loss RF coplanar transmission lines on flexible elastomeric polydimethylsiloxane (PDMS) substrates was developed and devices realized using this process were used to determine the characteristics of PDMS as an RF substrate with uniform low-loss and low dielectric constant being measured. To demonstrate the capabilities of this elastomerbased RF platform, a micro-mechanical switch exploiting a pneumatic membrane valve was integrated with the PDMS RF transmission line. Repeatable switching was observed with greater than $20 \mathrm{~dB}$ suppression in the 'off' state and minimal degradation of the transmission line characteristics in the 'on' state being achieved over a multi-octave $2-20 \mathrm{GHz}$ bandwidth. These valve-integrated transmission lines had insertion loss $0.16 \mathrm{~dB} \mathrm{~mm}^{-1}$ at $20 \mathrm{GHz}$. This proof-of-concept device represents a novel combination of the areas of micro-pneumatics, flexible electronics, and broadband microwave devices with excellent RF properties, low-interference, bias-free pneumatic switching and relatively simple fabrication.
\end{abstract}

Index Terms-Pneumatic switching, RF devices, Flexible electronics, PDMS, Coplanar waveguides

\section{INTRODUCTION}

Flexible electronics as an emerging technology builds on widely available microfabrication processes, combined often with soft lithography and nano-imprint techniques [1], [2], and has generated significant interest as it enables circuit functionalities to be employed on non-planar and conformal surfaces. Flexible devices have been realized on a variety of plastic and elastomeric substrates such as polyethylene [3], polyimide or Kapton [4], parylene [5], and polydimethylsiloxane (PDMS) [6], [7]. Rather than being limited to electronics applications such as light emitting diodes [8], these devices are increasingly capturing the

Manuscript received February 09, 2012. SS, MB, and TGN acknowledge Australian Post-Doctoral Fellowships from the Australian Research Council through Discovery Projects DP110100262, DP1092717, and DP1096153, respectively.

The authors are with the Functional Materials and Microsystems Research Group and Metamaterials Research Group, School of Electrical and Computer Engineering, RMIT University, GPO Box 2476, Melbourne, Victoria 3001, Australia (e-mail: sharath.sriram@gmail.com) attention of researchers in radio frequency (RF) technologies and metamaterials physics, driven by the pliable, conformal, and stretchable characteristics of elastomeric substrates [4][10].

Examples of RF devices demonstrated on flexible device platforms include curved antennas [9], patch antennas and coupled line filters [10], frequency selective surfaces and metamaterials [4]-[6], microwave frequency switches [11], and stretchable microfluidic RF antennas [12].

The use of PDMS in flexible electronics is of particular interest as it is widely employed in microfluidic and lab-on-achip devices due to its low surface energy [1], [2]. To control the flow of fluids in these devices, valves are often incorporated to either isolate or actuate the liquid flow [13], with such valves operating on piezoelectric [14], electromagnetic [15], or pneumatic [16] principles. The integration of such valve-based flow has enabled microfluidic devices with significant complexity to be realized.

One of the most important devices being explored in radio frequency micro-electro-mechanical systems (RF MEMS) is the electro-mechanical switch. These switches must provide strong suppression of the transmission over multi-octave bandwidths, while also offering low actuation voltages such that systems of these switches could be effectively realized [17], [18]. Methods to reduce the actuation voltage of switching for RF transmission lines by using complementary metal oxide semiconductor MEMS (CMOS-MEMS) techniques have been widely explored, but these solutions must often compromise on the quality of the RF device in both switching states (on and off) in order to achieve low-voltage actuation [19].

In this work, we report on the combination of PDMS-based RF electronic devices and pneumatic valves to achieve a pneumatically-switched RF coplanar waveguide. PDMS has stable high frequency dielectric properties and low loss [20], while pneumatic valve technology offers a non-electronic form of control of RF devices eliminating electromagnetic interference and parasitic effects caused by bias lines. The outcomes present opportunities for pneumatically-switched addressable RF devices and elements. Devices incorporating such pneumatic elements have potential switching times in the range of $10 \mathrm{~ms}$ [21]. These can be utilized to control RF transmission in order to potentially activate or disable RF 
devices such as antenna and metamaterials [22]. This will enable a new category of switchable and variable antennas and metamaterial arrays.

\section{FleXible Microelectrodes ON ELASTOMERIC SUBSTRATES}

To begin this investigation, we present a method for waferscale micro-fabrication of patterned conductors. This technique serves as a platform for the realization of flexible RF micro-devices to be discussed in the following section. The technique was developed to be compatible with standard silicon-based micro-fabrication processes [6], [23], [24]. A schematic of the fabrication sequence is shown in Fig. 1. A solvent cleaned silicon substrate was coated with a thin layer of aluminum [Figs. 1(a) and 1(b)], which prevents permanent adhesion of the subsequently coated PDMS to the silicon. A uniform layer of PDMS is spin-coated onto these substrates with its thickness in the range of 100 to $250 \mu \mathrm{m}$ with the exact thickness controlled by spin speed depending on the application [Fig. 1(c)] (see supplemental material, available at http://ieeexplore.ieee.org).

A conductive metal layer is deposited on these substrates by electron beam evaporation [Fig. 1(d)], followed by photolithography and wet chemical etching to pattern the conductors [Fig. 1(e)]. Finally, the entire PDMS layer is peeled off the silicon substrate, to realize free-standing and flexible elastomeric substrate with metal patterns [Fig. 1(f)]. The use of aluminum on the silicon substrate ensures a $100 \%$ yield in the last step, without which the PDMS layer tends to tear. Figure 2 shows an example of such a micro-fabricated device, where the PDMS substrate layer is $100 \mu \mathrm{m}$ thick and the metal conductors are defined by a gold-chromium combination about $200 \mathrm{~nm}$ thick. Patterned conductor strips with pads can be seen in Fig. 2(a), while Fig. 2(b) depicts the flexible characteristics of the elastomeric PDMS substrate.
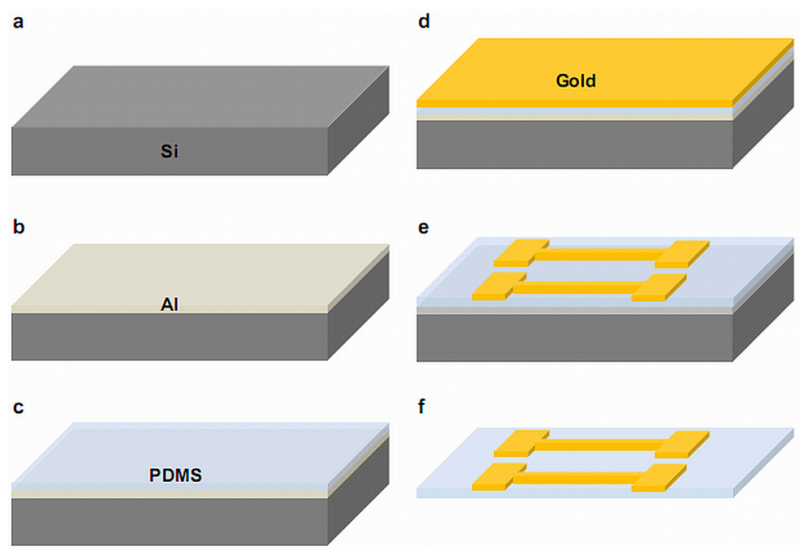

Fig. 1. Schematic of the fabrication sequence for flexible elastomeric microdevices: (a) pre-cleaned substrate, (b) deposition of $20 \mathrm{~nm}$ aluminum, (c) spin coating of PDMS, (d) deposition of metal layers, (e) patterning of metal layers, and (f) release of the flexible device from the substrate.

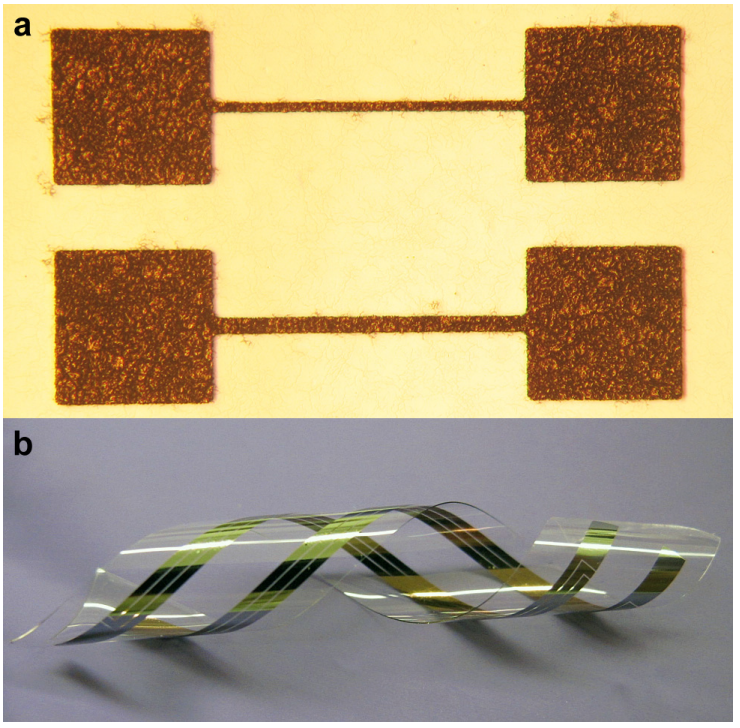

Fig. 2. Examples of fabricated elastomeric devices: (a) Optical micrograph of straight resistors and (b) flexed and twisted elastomeric device [24].

These results demonstrate that it is possible to use the described technique to realize electrodes with lines as narrow as a few microns and also with gaps in the same order. Simple conductivity testing has indicated that the devices retain their low resistance under bending and deformation.

\section{COPLANAR WAVEGUIDES ON PDMS}

Having demonstrated a technique that enables realization of micro-scale electrode structures on PDMS, we now utilize this technique to realize an RF transmission line in the form of a coplanar waveguide (CPW) and use this structure to characterize the broadband RF properties of the flexible PDMS substrate.

\section{A. Design and Fabrication}

The CPWs are relatively simple RF structures consisting of a central conductor and two symmetric adjacent ground planes (Fig. 3). Such CPW structures are travelling wave transmission lines which exhibit very uniform impedance, phase delay, and attenuation over ultra-broad bandwidths, and thus, can be used to accurately determine the RF properties of the dielectric substrates on which they are realized. For this work, the CPWs were designed to operate up to the RF frequency of $20 \mathrm{GHz}$. The width of the center conductor line and the gaps were calculated using standard design principles to achieve an impedance of $50 \Omega$ [25]. A design constraint was placed on the distance between the midpoint of the center conductor and edge of the ground planes to be less than $400 \mu \mathrm{m}$ (based on the pitch of the RF probes used for testing). For the initial design, an approximate value of 2.55 was chosen for the dielectric constant of PDMS, being at the midpoint of 2.3-2.8 quoted as the dielectric constant for this material [20]. The resulting design had a central conductor width of $600 \mu \mathrm{m}(w)$ and symmetric gaps of $60 \mu \mathrm{m}(\mathrm{g})$, as shown in Fig. 3. 


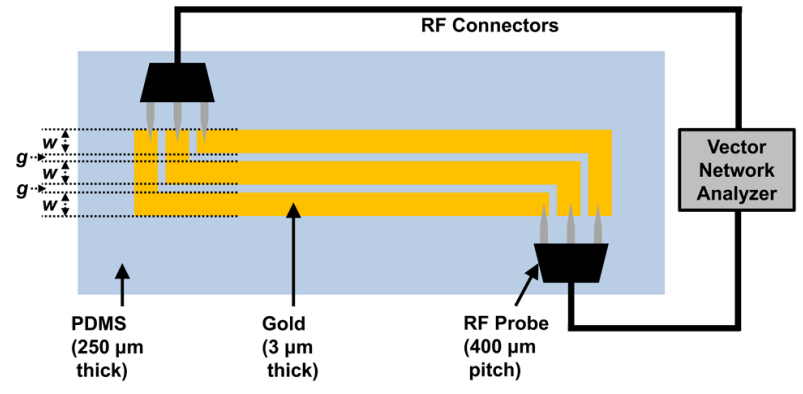

Fig. 3. Schematic of a coplanar waveguide showing its geometry and RF testing arrangement.

The thickness of the gold was set to $3 \mu \mathrm{m}(t)$ to ensure that it exceeded several skin depths at microwave frequencies [25]. The length $L$ of the CPW transmission lines varied from 10 to $40 \mathrm{~mm}$ (in $10 \mathrm{~mm}$ steps) to enable the accurate extraction of PDMS dielectric properties. Using these design parameters, elastomer-based CPW transmission lines were fabricated using the process in Section II (and Fig. 1). The PDMS layer was $250 \mu \mathrm{m}$ thick to improve handling and stability during RF probing and measurements. The metal deposition in this case was performed by DC magnetron sputtering to attain $3 \mu \mathrm{m}$ thick gold films.

The thickness of the gold and the final stage of release of the flexible PDMS device from the silicon substrate [Fig. 1(f)] resulted in surface micro-cracking of gold surface Fig. 4(a). Such micro-cracking only slightly increases the electrode resistance, with the effect of this determined during $\mathrm{RF}$ characterization. It should be noted that the micro-cracking enables the electrode structures to return to original resistance state after flexing or stretching, as the cracks allow the metal layers to coalesce. Further atomic force microscopy (AFM) characterization of the gold layers was also carried out. A typical result is presented in Fig. 4(b). These results highlight the textured surface of gold arising from the thermal mismatch between gold and PDMS [26]. This results in micro-scale wrinkles and macro-scale near-periodic peaks and troughs. The micro-cracks appear at these troughs and are found to be $\sim 1.3 \mu \mathrm{m}$ deep and $<0.5 \mu \mathrm{m}$ wide. These surface micro-cracks relate to $\sim 40 \%$ of the gold thickness indicating a significant portion of the gold that is greater than a few skin depths remains intact. The ability of the realized $\mathrm{CPW}$ to conform and flex is illustrated in Fig. 4(c).

\section{B. Characterization of the CPWs and Extraction of the RF Properties of PDMS}

The fabricated CPW lines were measured to estimate the dielectric constant and dielectric losses of the PDMS substrate. The CPWs under test were adhered to a glass slide to provide a rigid platform for probing and a vector network analyzer (VNA) was used in combination with high precision RF probes (40A-GSG-400-Q Picoprobe, GGB Industries, Inc.) as schematically depicted in Fig. 3.

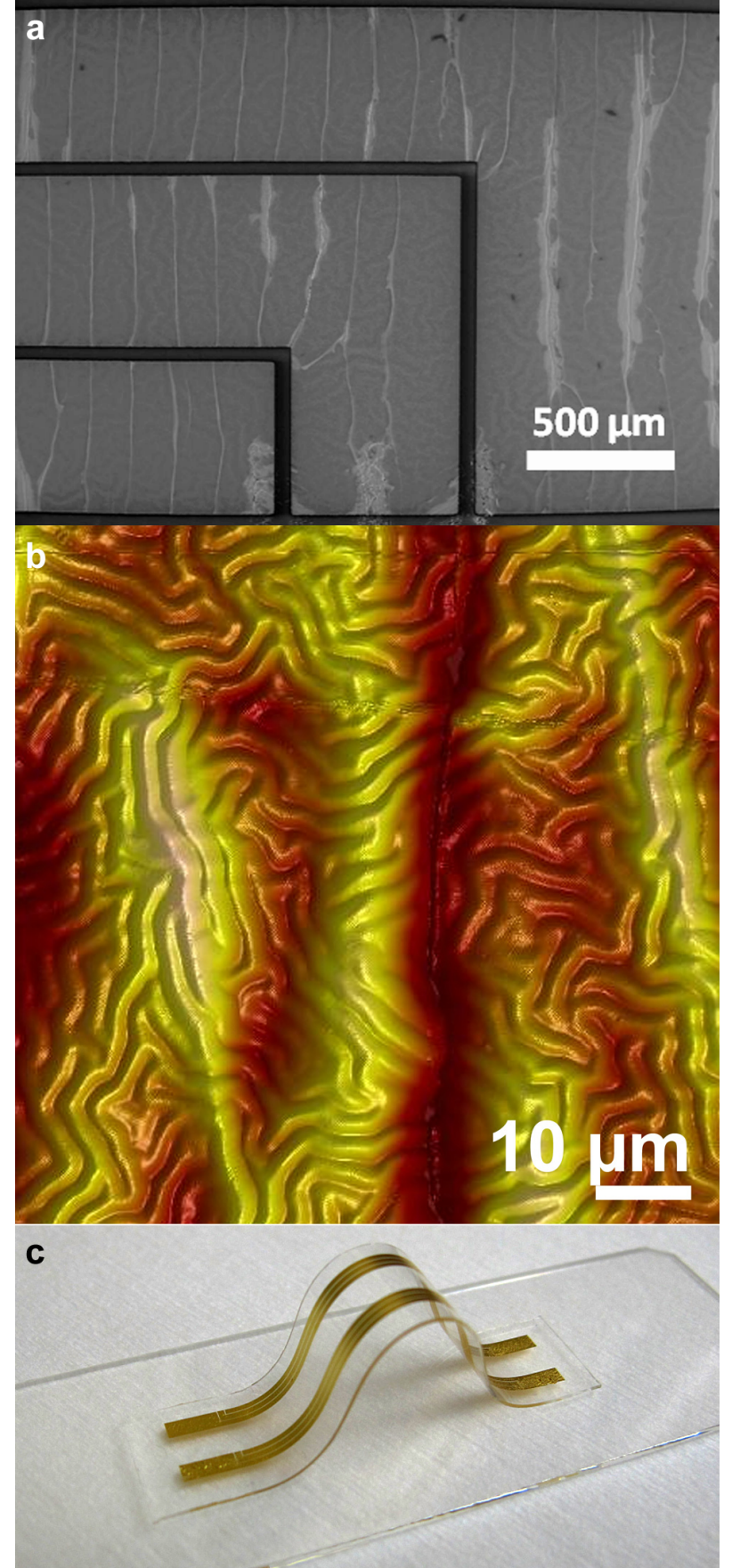

Fig. 4. Fabricated elastomeric coplanar waveguides utilizing $3 \mu \mathrm{m}$ thick gold layers: (a) Electron micrograph of micro-cracked morphology, (b) atomic force microscope scan showing surface micro-cracks, and (c) $40 \mathrm{~mm}$ long gold CPW electrodes (with dimensions as shown in Fig. 3) remain conductive when flexed as shown.

CPWs with four different lengths $(10,20,30$, and $40 \mathrm{~mm})$ were measured and the voltage transmission line response from Port 1 to Port $2\left(S_{21}\right)$ was recorded. The transmission properties for these four measurements are presented in the supplemental material as Fig. S1 available at http://ieeexplore.ieee.org. From the phase responses of the 


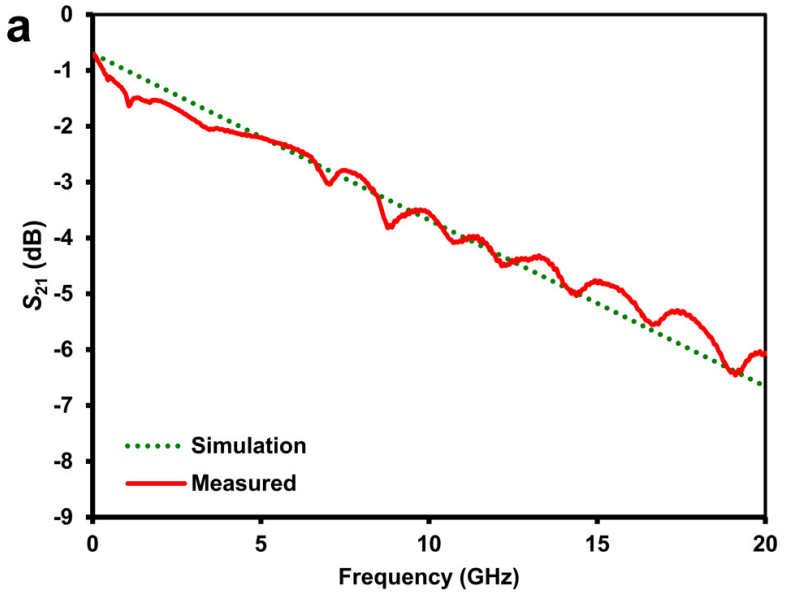

Fig. 5. Comparison of simulated and measured RF characteristics of a $40 \mathrm{~mm}$ long elastomeric CPW: (a) insertion loss and (b) return loss.

measured transmission response $\left(S_{21}\right)$, the RF effective index $\left(N_{\mathrm{m}}\right)$ of the CPW's was estimated to be in the range 1.3804 to 1.4217 with average RF effective index of 1.396. To estimate the RF dielectric constant of PDMS, the CPW electrode structure was simulated using a two dimensional finite element method (FEM) model [27]. The simulations were carried out for substrate dielectric constant ranging from 2.2 to 3.0 (see Fig. S2 available at http://ieeexplore.ieee.org.). From the FEM simulation results and the measured CPW RF effective index, the dielectric constant of PDMS was estimated to be 2.72 which is slightly higher than our initial approximation, but within the range of the reference data [20].

Figures 5(a) and 5(b) show the measured transmission $\left(S_{21}\right)$ and reflection $\left(S_{11}\right)$ response of one particular CPW with length of $40 \mathrm{~mm}$. Ripples can be observed in Fig. 5(a), as a by-product of minor impedance mismatch arising from the difference in ideal PDMS dielectric properties to the true dielectric properties. This minor mismatch is to be expected, as one of the aims of this process is to determine the true dielectric properties starting with ideal characteristics. Since the reflection response of Fig. 5(b) remains below $10 \mathrm{~dB}$ at all frequencies, it can be concluded that the impedance of the transmission line remains close to $50 \Omega$ over the entire bandwidth. The transmission response attenuates across the band with approximately $5.5 \mathrm{~dB}$ additional loss at $20 \mathrm{GHz}$. This loss is high, but not excessively so. The components of loss will be conductor loss due to the finite conductivity of the gold electrodes and the dielectric losses of the substrate. It may also be expected that the micro-cracks evident in figure $4 \mathrm{a}$ will contribute some additional resistive losses. The loss will have the form:

$$
S_{21} \propto e^{\alpha L}
$$

where

$$
\alpha(f)=\alpha_{\mathrm{c}} \sqrt{f}+\alpha_{\mathrm{d}} f
$$

with $\alpha_{\mathrm{c}}$ and $\alpha_{\mathrm{d}}$ are the loss coefficients due to finite conductivity of the electrodes and the dielectric loss of the substrate respectively. The loss due to the conductivity of the

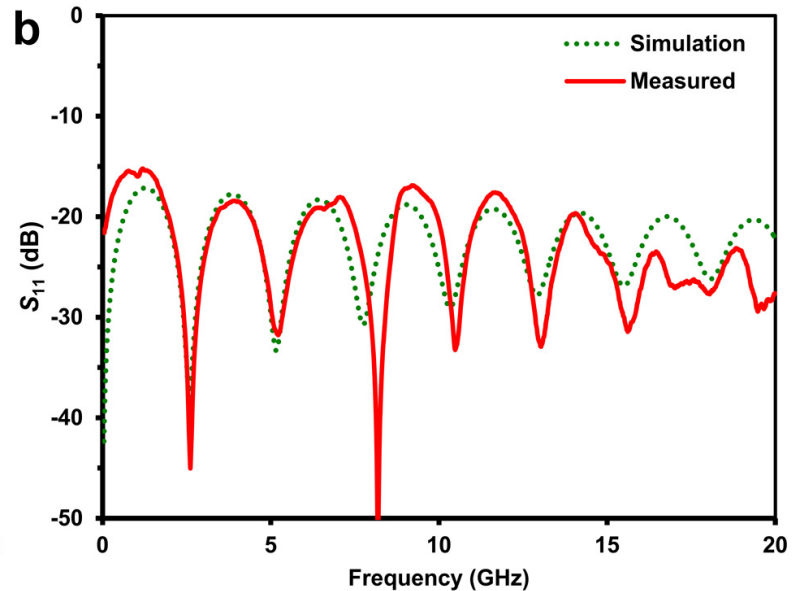

electrodes themselves can be readily calculated using the $2 \mathrm{D}$ FEM simulation results and a perturbation approach. The conductor loss coefficient $\alpha_{c}$ was calculated to be $0.033 \mathrm{~dB} \mathrm{~cm}^{-1} \mathrm{GHz}^{-0.5}$. If conductor loss was dominant, the transmission response would drop characteristically as the square root of frequency, however the response in Fig. 5(a) appears quite linear with frequency. The calculated conductor loss would also contribute significantly less attenuation than that observed in Fig. 5(a) and thus it can be concluded that the attenuation of the CPW electrode is not dominated by conductor loss. If we assume that the additional loss is entirely due to the dielectric loss PDMS we can estimate the magnitude of this loss by adjusting the value of $\alpha_{\mathrm{d}}$ until we obtain a best fit to the data in Fig. 5(a). Using this method, the PDMS dielectric loss $\alpha_{\mathrm{d}}$ was estimated to be in the range of $0.055-0.065 \mathrm{~dB} \mathrm{~cm}^{-1} \mathrm{GHz}^{-1}$. To the authors' knowledge, this is the first determination of the RF propagation loss properties for PDMS in the frequency range up to $20 \mathrm{GHz}$ range. It should be noted that the impact of the micro-cracks observed in Fig. 4(a) must be considered as potential sources of loss, however the loss coefficient calculated above does represent an upper limit on the dielectric coefficient of PDMS.

The transmission and reflection coefficients simulated using these extracted parameters are also presented in Figs. 5(a) and 5(b). It is clear that these parameters do provide a good description of the RF performance of the transmission line.

\section{Pneumatic Switching of Coplanar Waveguide}

\section{A. Pneumatic Micro-Valves}

Having demonstrated the realization and characterization of a broadband travelling wave CPW electrode on a PDMS substrate, we can now explore the exploitation of the elastomeric properties of this substrate material. To illustrate the utility that PDMS offers for RF we chose to implement a RF switch based on a pneumatically-actuated membrane valve. The valve design was based on that of Unger et al. [21], with the design scalable to as small as $20 \mu \mathrm{m} \times 20 \mu \mathrm{m}$. This 
a

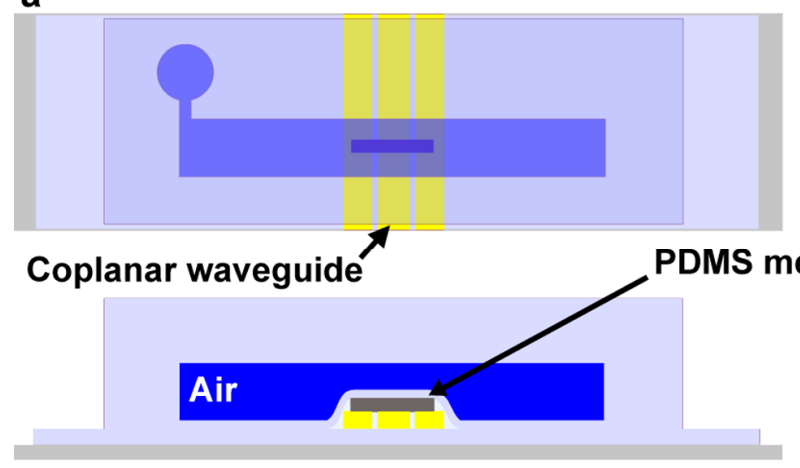

Switch Closed b

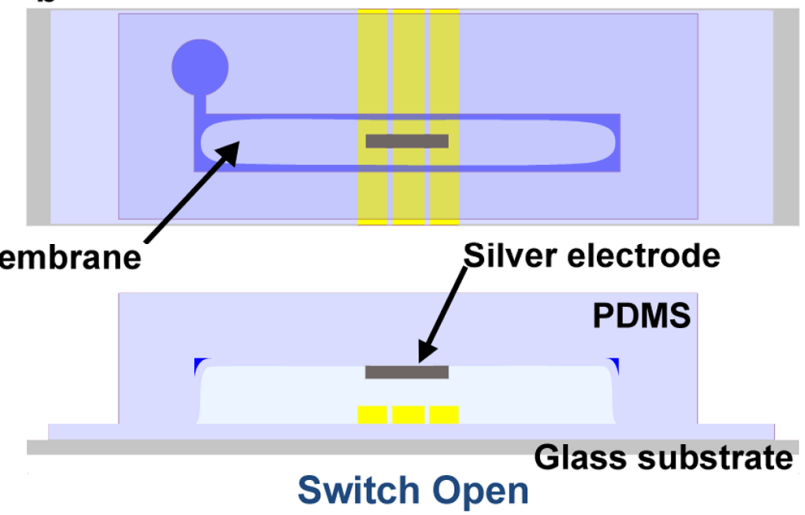

Fig. 6. Schematic with plan and cross-section views depicting the operation of the pneumatic RF switch in (a) closed state and (b) open state. An animated video of the operation is provided in the supplemental information (Video S1).

valve consisted of two bonded PDMS layers: a thick layer containing the control channel to enable the valve air pressure to be adjusted and a thin elastomeric membrane which is actuated by differential air pressure across the membrane.

These multilayer valves were realized using a combination of soft lithography and bonding techniques [21]. A thin metal electrode (silver epoxy) was defined on top of the membrane using an imprinting process. Full details of the fabrication process used to realize the micro-valve and the imprinted electrode are provided in the supplemental material available at http://ieeexplore.ieee.org.

Figure 6 presents an illustration of how this valve is operated as an RF switch. The valve structure is oriented such that the metalized membrane is placed face down over the CPW electrode. The low surface energy of the PDMS of both the valve and the CPW components is sufficient to bond these two components together. In the 'off' state [Fig. 6(a)], the air pressure in the control channel is made slightly positive in order to ensure good contact between the CPW electrodes and the shorting electrode on the membrane. In the 'on' state [Fig. 6(b)], the air in the control channel is evacuated, the membrane is thus retracted into the control channel cavity breaking the electrical contact with the CPW electrodes and removing the shorting electrode from the vicinity of the CPW. An animated video of the switching process is provided as supplemental material available at http://ieeexplore.ieee.org.

Figure 7 presents a photograph of the realized pneumatic switch assembled with the CPW electrode. The valve membrane dimensions $(10 \mathrm{~mm} \times 1 \mathrm{~mm})$ and channel depth $(80 \mu \mathrm{m})$ were chosen to ensure stable and repeatable switching performance (see Fig. S3 available at http://ieeexplore.ieee.org).The length of the shorting electrode on the PDMS membrane was in excess of $800 \mu \mathrm{m}$ to ensure that all three CPW conductors (the central conductor and the two outer ground planes) were electrically shorted when the shorting electrode was brought into contact. The fabricated pneumatic micro-valve (Section IVA) is placed in contact with the elastomeric CPW structure (Section IIIA). The silver conductor on the micro-valve membrane is positioned (using an optical microscope) such that it overlaps the three conductors of the CPW. The control channel was actuated and evacuated using a manual syringe as shown in Fig. 7(a).

\section{B. Testing of Pneumatic Switching}

Switching functionality was first tested under direct current (DC) conditions using a multi-meter, before proceeding to RF testing. This showed that slight positive pressure is required for the shorting electrode to make good electrical contact with the gold CPW electrodes [Fig. 6(a)]. After DC testing, the device was placed on an RF probe station as shown in Fig. 7(b).

The first stage of testing focused on determining whether the integration of the PDMS valve on top of the CPW had a detrimental effect to the RF signal transmission. Figure 8(a) presents the measured transmission coefficient of the original elastomeric CPW line (Fig. 5) to the measured transmission coefficient of the integrated CPW line and pneumatic switch set to the 'on' position by evacuating the control channel as in Fig. 6(b). The results show that the insertion loss increases slightly when the air region over the CPW is replaced with PDMS. This increased loss can be attributed to an increase in the effective index of the transmission line (resulting in higher conductor losses due to the longer duration spent interacting with the electrode); an increase in the characteristic impedance of the line and also additional dielectric loss due to the increased interaction with additional PDMS valve layer. To illustrate the utility of our extracted PDMS parameters, the transmission characteristics of the transmission line was modeled using FEM and the extracted parameters from the previous section both with and without the pneumatic valve layer in place and the simulated results are presented in Fig. 8(b). Excellent agreement between simulated and measured data is evident, supporting the accuracy of our extracted dielectric loss parameter for PDMS.

The switch was then set to the 'off' position by returning air to the control channel with slightly positive pressure as in Fig. 6(a) and the transmission characteristics were again measured using the RF probe station [Fig. 7(b)] with the measured results presented in Fig. 8(c). With the shorting electrode in contact with the CPW, the measured transmission drops by $20 \mathrm{~dB}$ with respect to the un-shorted case in Fig. 8(a). This extinction of the transmission is effective over the entire $20 \mathrm{GHz}$ bandwidth indicating excellent contact 


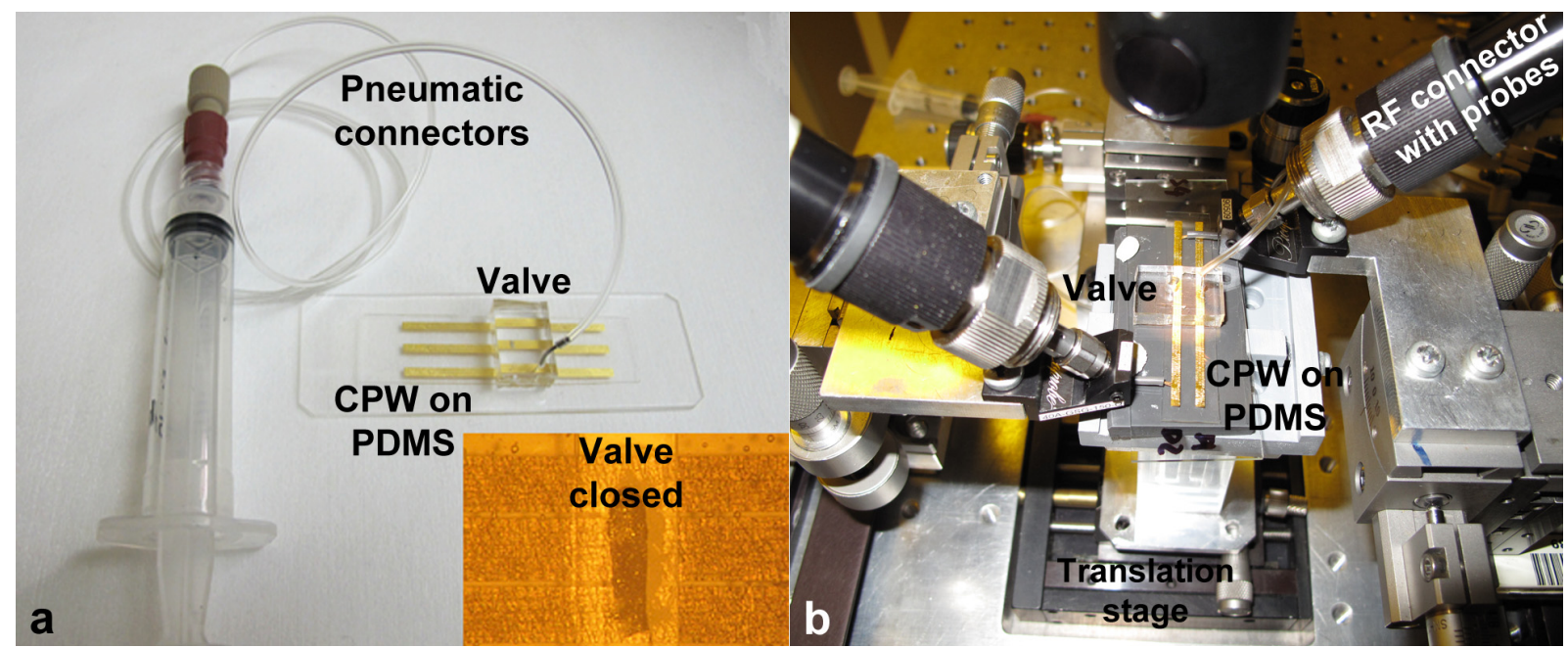

Fig. 7. (a) Integrated pneumatic RF switch with valve placed on a CPW transmission line, with inset showing an optical micrograph of the closed state. The valve dimensions are shown in Fig. S3. (b) Photograph of the RF testing arrangement with the integrated device.
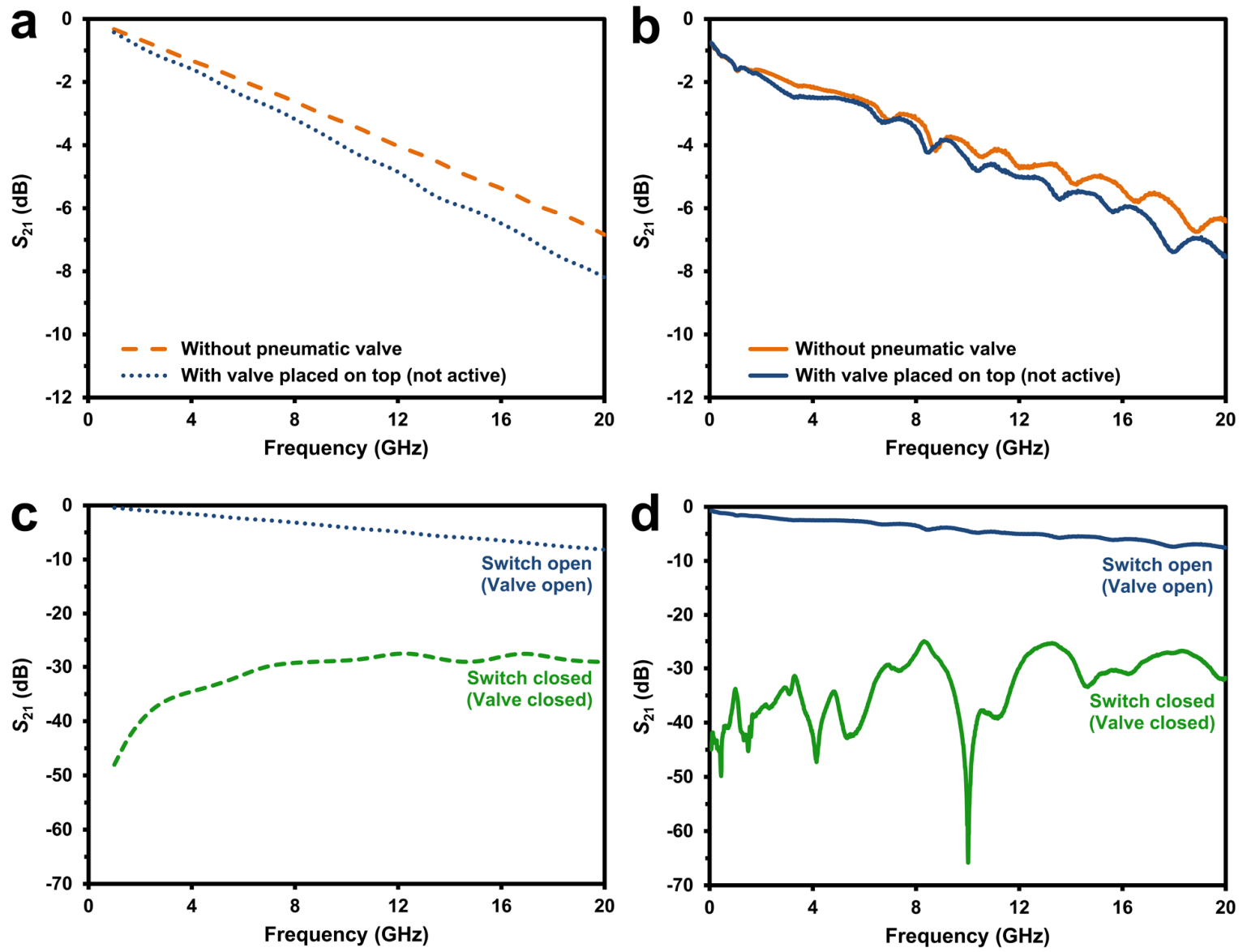

Fig. 8. Measured and simulated transmission data presented in (a) and (b), respectively, demonstrates the effect of placing the valve (in its inactive, open state) on top of the CPW line. A slight increase in loss is observed. Measured and simulated data presented in (c) and (d), respectively, characterizes the pneumatic switching. A minimum $20 \mathrm{~dB}$ difference can be observed between the valve open (full transmission) and closed (nil transmission) states.

is achieved between the CPW and the pneumatically actuated shorting electrode. The RF signal that is not transmitted is in fact reflected back to Port 1 . This reflection coefficient was also measured and is included in the supplemental material
(Fig. S5 available at http://ieeexplore.ieee.org). The expected transmission behavior in the off state was also modeled using FEM and our extracted PDMS parameters and these simulated results are presented in Fig. 8(d). 
Again, excellent agreement is evident between the measured results and simulated predictions, further demonstrating the utility of our extracted PDMS parameters. These results show that pneumatics can be used successfully to achieve a broad band, high extinction RF switch which is free from electromagnetic interference due to the absence of bias lines.

These devices with potential switching times in the range of $10 \mathrm{~ms}$ [21] can be utilized to alter the state of RF transmission and/or potentially activate or disable RF devices such as antenna and metamaterials [22]. The excellent agreement between our measured and simulated results also indicate that our extracted parameters can be used to reliably model and design RF devices that utilize PDMS as an pneumatic electromechanical platform.

\section{CONCLUSIONS}

We have demonstrated the integration of flexible electronics, microfluidic technology, and $\mathrm{RF} /$ microwave components to demonstrate the first pneumatically-switched RF micro-devices. A process for high yield flexible device fabrication is presented, along with the technical adaptations required for the fabrication of RF micro-devices (coplanar waveguides). A travelling wave electrode realized using this technique has been used to extract the RF properties of the PDMS material. An RF switch based on pneumatic valves were designed using these extracted PDMS properties and were fabricated and integrated with these coplanar waveguides. The integration of this pneumatic switch had only minor impact on the RF properties of the CPW in the 'on' state and provided $20 \mathrm{~dB}$ extinction of the transmission in the 'off' state. Excellent agreement was achieved between the simulated design and the measured results highlighting the utility of our extracted RF characteristics of the PDMS material used.

Future work will examine designs that reduce losses of the CPW transmission lines. It will also explore changes in RF transmission when the structures are flexed or bent. Such elastomer-based devices can be used to realize more sophisticated networks of pneumatically controlled RF devices with particular emphasis on applications in reconfigurable circuits and even metamaterials.

\section{REFERENCES}

[1] Y. Xia and G. M. Whitesides, "Soft lithography," Ann. Rev. Mater. Sci. vol. 28, no. 1, pp. 153-184, Aug. 1998.

[2] J. A. Rogers and R. G. Nuzzo, "Recent progress in soft lithography," Mater. Today, vol. 8, no. 2, pp. 50-56, Feb. 2005

[3] Y. Li, L. W. Tan, X. T. Hao, K. S. Ong, F. Zhu, and L. S. Hung, "Flexible top-emitting electroluminescent devices on polyethylene terephthalate substrates," Appl. Phys. Lett., vol. 86, no. 15, art. no. 153508, Mar. 2005

[4] H. Tao, A. C. Strikwerda, K. Fan, C. M. Bingham, W. J. Padilla, X. Zhang, and R. D. Averitt, "Terahertz metamaterials on free-standing highly-flexible polyimide substrates ," J. Phys. D: Appl. Phys., vol. 41, no. 23 , art. no. 232004 , Nov. 2008

[5] X. Liu, S. MacNaughton, D. B. Shrekenhamer, H. Tao, S. Selvarasah, A. Totachawattana, R. D. Averitt, M. R. Dokmeci, S. Sonkusale, and W. J. Padilla, "Metamaterials on parylene thin film substrates: Design, fabrication, and characterization at terahertz frequency," Appl. Phys. Lett., vol. 96, no. 1, art. no. 011906, Jan. 2010.

[6] I. E. Khodasevych, C. M. Shah, S. Sriram, M. Bhaskaran, W. Withayachumnankul, B. S. Y. Ung, H. Lin, W. S. T. Rowe, D. Abbott, and A. Mitchell, "Elastomeric substrates for terahertz metamaterials," Appl. Phys. Lett., vol. 100, no. 6, art. no. 061101, Feb. 2012.

[7] J. A. Rogers, T. Someya, and Y. Huang, "Materials and Mechanics for Stretchable Electronics," Science, vol. 327, no. 5973, pp. 1603-1607, Mar. 2010.

[8] J. M. Lee, J. W. Choung, J. Yi, D. H. Lee, M. Samal, D. K. Yi, C. H. Lee, G. C. Yi, U. Paik, J. A. Rogers, and W. I. Park, "Vertical Pillar-Superlattice Array and Graphene Hybrid Light Emitting Diodes," Nano Lett., vol. 10, no. 8, pp 2783-2788, Jul. 2010.

[9] J. J. Adams, E. B. Duoss, T. F. Malkowski, M. J. Motala, B. Y. Ahn, R. G. Nuzzo, J. T. Bernhard, and J. A. Lewis, "Conformal Printing of Electrically Small Antennas on Three-Dimensional Surfaces," Adv. Mater., vol. 23, no. 11, pp. 1335-1340, Jan. 2011.

[10] N. Tiercelin, P. Coquet, R. Sauleau, V. Senez, and H. Fujita, "Polydimethylsiloxane membranes for millimeter-wave planar ultra flexible antennas," J. Micromech. Microeng., vol. 16 no. 11, art. no. 2389, Nov. 2006.

[11] H.-C. Yuan, G. Qin, G. K. Celler, and Z. Ma, "Bendable high-frequency microwave switches formed with single-crystal silicon nanomembranes on plastic substrates," Appl. Phys. Lett., vol. 95, no. 4, art. no. 043109, Jul. 2009.

[12] M. Kubo, X. Li, C. Kim, M. Hashimoto, B. J. Wiley, D. Ham, and G. M. Whitesides, "Stretchable Microfluidic Radiofrequency Antennas" Adv. Mater., vol. 22, no. 25, pp. 2749-2752, Apr. 2010.

[13] J. Melin and S. R. Quake, "Microfluidic Large-Scale Integration: The Evolution of Design Rules for Biological Automation," Ann. Rev. Biophys. Biomol. Struct., vol. 36, pp. 213-231, Jun. 2007.

[14] I. Fazal and M. C. Elwenspoek, "Design and analysis of a high pressure piezoelectric actuated microvalve," J. Micromech. Microeng., vol. 17, no. 11 , art. no. 2366 , Nov. 2007.

[15] A. Gaspar, M. E. Piyasena, L. Daroczi, and F. A. Gomez, "Magnetically controlled valve for flow manipulation in polymer microfluidic devices," Microfluid. Nanofluid., vol. 4, no. 6, pp. 525-531, Jun. 2008.

[16] W. H. Grover, R. H. C. Ivester, E. C. Jensen, and R. A. Mathies, "Development and multiplexed control of latching pneumatic valves using microfluidic logical structures," Lab Chip, vol. 6, pp. 623-661, Apr. 2006.

[17] N. Lorphelin, R. Robin, A. S. Rollier, S. Touati, A. Kanciurzewski, O. Millet, and K. Segueni, "Simulation and optimization of a totally free flexible RF MEMS switch," J. Micromech. Microeng., vol. 19, no. 7, art. no. 074017 Jul. 2009.

[18] T.-Y. Chao, M. C. Hsu, C.-D. Lin, and Y. T. Cheng, "SU-8 serial MEMS switch for flexible RF applications," J. Micromech. Microeng., vol 21 , no. 2 , art. no. 025010, Jan. 2011.

[19] C.-L. Dai and J.-H. Chen, "Low voltage actuated RF micromechanical switches fabricated using CMOS-MEMS technique," Microsyst. Technol., vol. 12, no. 12, pp. 1143-1151, Oct. 2006.

[20] Sylgard 184 Silcione Elastomer Kit Product Data Sheet, Dow Corning Corporation. Available online at: http://www.dowcorning.com/applications/search/default.aspx?r=131en

[21] M. A. Unger, H. P. Chou, T. Thorsen, A. Scherer, and S. R. Quake, "Monolithic microfabricated valves and pumps by multilayer soft lithography," Science, vol. 288, no. 5463, pp. 113-116, Apr. 2000.

[22] I. E. Khodasevych, W. S. T. Rowe, and A. Mitchell, "Reconfigurable fishnet metamaterial using pneumatic actuation," Prog. Electromag. Res. $B$, vol. 32, pp. 57-70, 2012.

[23] S. P. Lacour, S. Wagner, Z. Huang, and Z. Suo, "Stretchable gold conductors on elastomeric substrates," Appl. Phys. Lett., vol. 82, no. 15, art. no. 2404, Feb. 2003.

[24] C. M. Shah, S. Sriram, M. Bhaskaran, and A. Mitchell, "Large area metal-silicone flexible electronic structures," in Proceedings of the 2010 Conference on Optoelectronic And Microelectronic Materials and Devices, pp. 187-188, IEEE, Canberra, 2010.

[25] R. N. Simons, Coplanar waveguide circuits components and systems, Wiley-IEEE Press, 2001.

[26] N. Bowden, S. Brittain, A. G. Evans, J. W. Hutchison, and G. M. Whitesides, "Spontaneous formation of ordered structures in thin films 
of metal supported on an elastomeric polymer," Nature, vol. 393, no. 6681, pp. 146-149, May 1998.

[27] J.-M. Jin, The finite element method in electromagnetics, John Wiley \& Sons, 1993.

Charan M. Shah received his Master of Engineering (Microelectronics) from RMIT University in 2009. He worked on the bulk micromachining of silicon to realize silicon nitride membranes and coplanar waveguides and on the fabrication of V-grooves arrays for optical fiber loading. He is currently undertaking a Ph.D. on the realization of tunable metamaterials at millimeterwave frequencies, incorporating the development of fabrication methods and techniques for flexible electronic devices.

Sharath Sriram (S'03-M'12) received his Doctor of Philosophy (Ph.D.) degree RMIT University, Melbourne, Australia, in 2009. He specializes in the deposition and materials characterization of complex functional oxide thin films for electronic applications. He has developed optimized deposition processes for piezoelectric thin films and simplified techniques for their characterization. His current research interests include piezoelectrics, Raman scattering, electrically tunable behavior in oxides, and flexible electronic and electromagnetic devices. He is currently undertaking an Australian PostDoctoral Fellowship funded by the Australian Research Council.

Madhu Bhaskaran (S'03-M'12) received the Doctor of Philosophy (Ph.D.) degree from the RMIT University, Melbourne, Australia, in 2009. She is the recipient of an Australian Post-Doctoral Fellowship 2010-2013 from the Australian Research Council. She has exceptional skills in silicon-based fabrication techniques and thin-film patterning for the development of devices. She is adept at thin-film characterization using a variety of spectroscopy, microscopy, and diffraction techniques. Her current research interests include piezoelectric energy harvesting and characterization of nanostructured materials.

Mahyar Nasabi (GSM'07-12) received his Master of Engineering (Electronics) from RMIT University in 2008. He worked on the micro-valve technologies in microfluidics to realize on-chip control for lab-on-a-chip devices while he was working as a Research Officer at the Microelectronics and Materials Technology Centre at RMIT University. He is currently undertaking a Ph.D. on the realization of a lab-on-a-chip platform for investigating blood clotting in pulsed flow.

Thach Giang Nguyen received his received the B.Eng. and Ph.D. degrees in communication engineering from RMIT University in 1998 and 2006, respectively. From 1999 to 2002, he was a Design Engineer with Vietnam Telecommunications Technology Co. (VITECO), Vietnam Post and Telecommunications Group (VNPT), Hanoi, Vietnam. His research interests are investigation of specialized optical modulators and modeling of integrated photonic devices. He is a current recipient of an Australian Post-Doctoral Fellowship investigating lateral leakage in thin ridge silicon waveguides.

Wayne Rowe (S'98-A'02-M'12) received the B.Eng. (honors) degree and the Ph.D. degree in communication engineering from RMIT University, Melbourne, Australia, in 1997 and 2002, respectively. In 2002, he commenced employment as a Research Fellow affiliated with both the Australian Photonics Cooperative Research Centre and the Microelectronics and Materials Technology Centre. He accepted a Lecturer position within the School of Electrical and Computer Engineering at RMIT University, in 2004. He has performed consulting work for numerous companies including: the Australian Army Engineering and Technology Agency, the Defense and Sciences Technology Organization, and British Aerospace Australia (BAE) Systems. His research interests include printed antennas, integrated antenna/microwave/photonic modules, photonic signal processing and optically distributed microwave/millimeter wave systems.

Arnan Mitchell received his Ph.D. from RMIT University in 1999. He is the leader of the Microplatforms Research Group within the Platform Technologies Research Institute at RMIT University and a Chief Investigator of the ARC Centre of Excellence in Ultrahigh bandwidth Devices for Optical Systems (CUDOS). Prof. Mitchell works closely with a large team of early career researchers pursuing research in a broad range of fields including integrated optics, lab-on-a-chip platforms, microwave photonics, RF microplatforms, functional materials, and microsystems. 